\title{
CORRELAÇÃO ENTRE ÁRVORES NA CALÇADA E CONSUMO DE ENERGIA ELÉTRICA
}

\author{
CORRELATION BETWEEN TREES IN SIDEWALK AND ELECTRICITY \\ CONSUMPTION \\ Wellington Jorge Cavalcanti Lundgren ${ }^{1}$; Thialla Laranjeira Amorim²; Giovanna Alencar Lundgren³
}

\section{RESUMO}

Nesse artigo foi verificada a correlação da copa e altura da árvore com o consumo de energia elétrica para famílias de baixo poder aquisitivo. Foi verificada a correlação do número de residentes com o consumo de energia elétrica. As casas foram divididas em dois subgrupos: com dois ou menos moradores e mais de dois moradores, cada subgrupo foi novamente dividido em com árvores na calçada e sem árvores na calçada. Foi verificado que o número de moradores tem correlação positiva com o consumo de energia elétrica $(r=0,60)$. O consumo de casas com dois ou menos moradores difere estatisticamente do consumo de casas com mais de dois moradores. Para casas com dois ou menos moradores o aumento de um metro na altura da árvore gera uma tendência de diminuição do consumo de energia elétrica de $13 \mathrm{kw} / \mathrm{mês}$ e o aumento de um metro no diâmetro da copa gera uma tendência de diminuição no consumo de $14 \mathrm{kw} / \mathrm{mês}$. Cada pessoa provoca uma tendência de aumento de consumo de aproximadamente $24 \mathrm{kw} / \mathrm{mês}$. Para casa com mais de dois moradores a presença de árvores na calçada não afeta significativamente o consumo de energia elétrica.

Palavras-chave: Altura da árvore; Copa da árvore; Número de residentes; Benefícios da Arborização.

\section{ABSTRACT}

In this article the correlation of the tree canopy and height was observed with the consumption of electricity for low income families. The correlation of the number of residents with electricity consumption has been verified. The houses were divided into two groups: less than three residents and more than two residents, each subgroup was further divided into with trees on the pavement and treeless on the sidewalk. It was found that the number of residents has positive correlation with the consumption of electricity, $r=0.60$. The consumption of households with less than three is statistically different from the consumption of households with more than two residents. For houses with less than three residents, the increase of one meter in height of the tree generates a tendency of decrease in electricity consumption of $13 \mathrm{kw} / \mathrm{month}$ and an increase of one meter in diameter canopy generates a downward trend in consumption of $14 \mathrm{kw} /$ month. Each person causes an increasing trend of consumption of approximately $24 \mathrm{~kW} / \mathrm{month}$. For homes with more than two residents the presence of trees on the sidewalk does not significantly affect the consumption of electricity.

Keywords: Tree height; Treetop; Number of residents; Benefits of afforestation.

Recebido em 03.12.2015 e aceito em 11.01.2015

1 Engenheiro Florestal, Dr. Professor da Universidade Federal Rural de Pernambuco - Unidade Acadêmica de Serra Talhada, Serra Talhada/PE. Email:wellingtonlundgren@yahoo.com.br

2 Bióloga pela Universidade Federal Rural de Pernambuco - Unidade Acadêmica de Serra Talhada, Serra Talhada/PE. Email: tiallaamorim@hotmail.com

3 Engenheira Agrônoma, mestranda em Agronomia (Horticultura), Universidade Estadual Paulista - Faculdade de Ciências Agronômicas, Botucatu/SP. Email: giolundgren@gmail.com 


\section{INTRODUÇÃO}

A população mundial que vivia no meio urbano até o ano de 2007 era em torno de 2,9 bilhões e com a perspectiva de ser em 2030 cinco bilhões de habitantes (MARTINE, 2007). Em 2015, estima-se que existam aproximadamente sete bilhões de habitantes no planeta.

O aumento da população de uma cidade implica na diminuição da vegetação existente no local, pois construção urbana leva invariavelmente a retirada da vegetação local. Esse fato foi constatado por Feitosa et al. (2011) em pesquisa realizada em Terezina - PI. Percebe-se, empiricamente, que muita vegetação nativa ainda será suprimida até a estabilização da população mundial.

Os benefícios que uma adequada arborização proporciona aos habitantes de aglomerados urbanos de qualquer porte já são bem conhecidos e estudados por diversos pesquisadores. Roppa et al. (2007) listaram e discutiram alguns dos benefícios, como diminuição da poluição atmosférica, da poluição sonora, aumento da retenção de carbono, interceptação da água da chuva diminuindo o risco de inundação, além do aumento da sensação de bem estar.

Kurihara et al. (2005) dividem os benefícios em três classes: ecológica, social e estética. Dentro dos benefícios já citados anteriormente, eles especificam, controle da temperatura e do vento, atração de pequenos animais silvestres e controle da erosão, todos esses inclusos na classe de benefícios ecológicos. Na classe social eles incluem, oportunidade de recreio, valorização cultural e histórica da vegetação. Para a classe estética a diversificação da paisagem e criação de espaços abertos.

Existem alguns estudos que verificam a correlação existente entre o consumo de energia elétrica e a arborização urbana. Velasco et al. (2011) tentaram correlacionar o número de árvores por residência de três diferentes zonas com o consumo de energia elétrica no município de São Paulo - SP, porém eles afirmam que não foi possível medir o consumo de energia elétrica e usou o número de aparelhos de ar condicionados não conseguindo diferença significativa, pois o número de aparelhos não diferiu entre as zonas.

Souza et al. (2009) correlacionaram a temperatura nas ilhas de calor com o consumo de energia elétrica no município de Bauru - SP. Os autores encontraram correlação positiva, contudo, na pesquisa não foi levado em conta a arborização urbana local. Levando-se em conta o trabalho de Velasco et al. (2011) em que foi verificado que quanto maior a área da arborização urbana, menor a temperatura do ar e maior a umidade do ar, de forma empírica pode-se concluir que a arborização diminui o consumo de energia elétrica. 
O objetivo da pesquisa foi verificar se a presença de árvores na calçada em frente a residência e a dimensão da copa e altura está correlacionada com o consumo de energia elétrica em moradias de baixo poder aquisitivo na cidade de Serra Talhada - PE.

\section{MATERIAL E MÉTODOS}

A pesquisa foi realizada na cidade de Serra Talhada - PE, localizada na latitude $7^{\circ} 59^{\prime}$ 9" S e longitude $38^{\circ} 17^{\prime} 45^{\prime \prime} \mathrm{W}$ (SUDENE, 1990). A cidade possui, segundo o IBGE (2010), cerca de 90.000 habitantes, tem clima quente e seco com temperatura máxima que pode chegar a $38^{\circ} \mathrm{C}$. A Figura 1 mostra a localização e fotos da rua onde foi realizada a pesquisa.

A rua Benício de Souza Ramos foi escolhida por ficar localizada no bairro Nossa Senhora da Penha de baixo poder aquisitivo onde as casas em sua maioria não ultrapassam a largura de cinco metros de frente, com calçadas estreitas e as casas não possuem varanda. Essa característica implica em afirmar que não existe uma área de arrefecimento da temperatura do ar da rua para a casa, ou seja, apenas uma parede separa a sala da casa da rua.

Nogueira et al. (2011) compararam a temperatura interna entre dois tipos de residências na cidade de Cascavel - PR, uma residência tradicional, outra com construção alternativa. Com termômetros em todos os aposentos das casas e mediram as temperaturas em vários horários diferentes ao longo do dia. Concluíram que a diferença entre os diversos aposentos da casa (quarto, sala, cozinha etc.), assim como os dois tipos de residências, não diferiram estatisticamente. Vale ressaltar que as duas casas não possuíam varandas.

Um questionário foi aplicado em todas as residências da rua Benício de Souza Ramos. Não entrou na pesquisa estabelecimentos comerciais.

Os seguintes dados foram coletados: número da casa, número de residentes, consumo de energia elétrica dos meses de setembro, outubro e novembro de 2013, quais eletrodomésticos existentes na residência, renda mensal do domicílio, altura da árvore em frente da casa, diâmetro da copa da árvore e se a casa está voltada para o oeste.

Os meses de setembro, outubro e novembro foram escolhidos por coincidirem segundo Assis et al. (2013), com o início dos meses mais quentes, além de não serem influenciados pelo consumo comumente aumentando no mês de dezembro devido às festas natalinas. Para a coleta dos dados sobre o consumo de energia foi solicitado ao morador a apresentação de uma conta de energia, que tem o histórico do consumo da residência. 

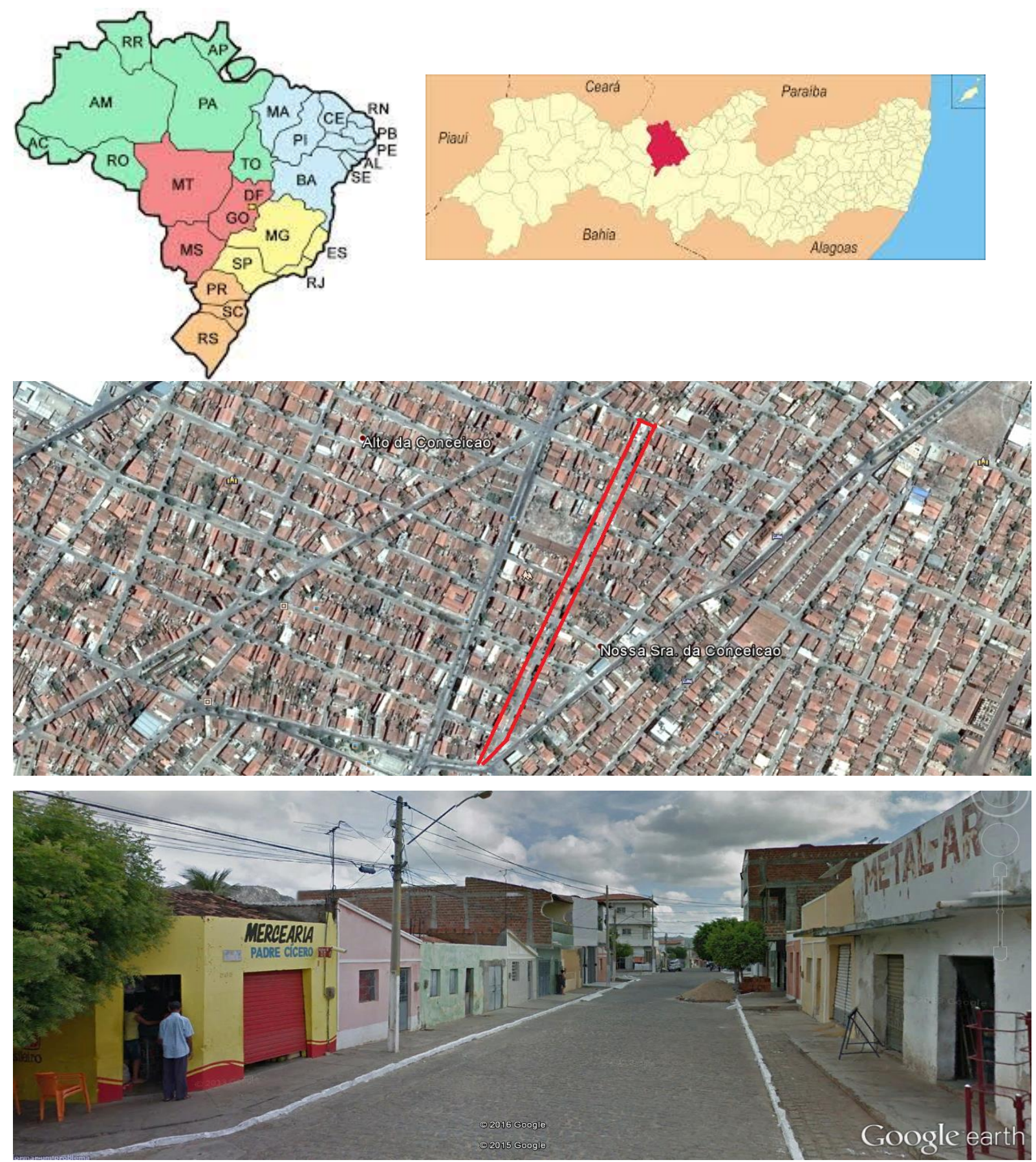

Fontes: Mapa de Wellber Drayton - Wikipédia. Mapa do Exército Brasileiro. Google Earth.

Figura 1. Localização da rua Benício de Souza Ramos na cidade de Serra Talhada - PE Figure 1. Location of Benicio de Souza Ramos Street in the city of Serra Talhada - PE

O diâmetro da copa foi medido com fita métrica simples sempre no sentido do acompanhamento da calçada e a altura foi estimada usando o mesmo procedimento descrito por Lundgren e Silva (2013).

$\mathrm{O}$ teste $\mathrm{F}$ foi utilizado para comparar as classes residenciais, com duas ou menos pessoas $x$ residências com mais de duas pessoas. Cada uma das classes residenciais foi subdividida em duas, residências com árvores na frente de casa e residências sem árvores na 
frente de casa e também comparadas pelo teste F. Também foram comparadas residências com frente para o oeste $\mathrm{x}$ residências com frente para o leste.

Foram construídos gráficos de colunas para a média de consumo das residências com frente para o oeste e para o leste, para o consumo médio de casas com e sem árvores na calçada e para residências com dois ou menos moradores e casas com mais de dois moradores. Também foram construídos gráficos de dispersão, calculada a correlação, construída uma equação de regressão e calculado o coeficiente de determinação para o número de residentes $x$ consumo de energia, para a altura da árvore $x$ consumo de energia $e$ diâmetro da copa $x$ consumo de energia.

Para cada uma das equações de regressão foram testados três modelos:

a) Linear simples $-Y=a X+b+e$

b) Exponencial $-Y=a E^{b X}+e$

C) Polinomial de segunda ordem $-Y=a X^{2}+b X+c+e$

Em que:

$Y=$ variável resposta

$\mathrm{X}=$ variável explicativa

$a, b, c=$ coeficientes da equação

$\mathrm{e}=$ erro do modelo

$\mathrm{E}=$ número de Euler

A escolha do modelo foi feita pelo maior valor do $\mathrm{R}^{2}$.

\section{RESULTADOS E DISCUSSÕES}

\section{a) Número de residentes $x$ Consumo de energia elétrica}

O consumo de energia de uma residência, segundo Shiu e Lam (2004) está diretamente correlacionado com diversos fatores, entre eles; número de pessoas que moram na residência, grau de crescimento econômico da população, temperatura, etc.

A Figura 2 mostra o gráfico de dispersão número de residentes $x$ consumo de energia elétrica e a linha do modelo ajustado.

A correlação entre o número de moradores e o consumo de energia foi de $r=0,60$. Quanto maior o número de pessoas residentes em uma casa, maior o consumo de energia da residência. A equação que melhor se ajustou aos dados foi a potencial, que forneceu $0 \mathrm{R}^{2}=$ 0,4759, $\left(Y=67,499 E^{0,6268 X}\right)$. 
Porém, a correlação de 0,60, entre o número de moradores e o consumo de energia, pode influir significativamente na hipótese do trabalho, que é a existência de correlação negativa entre as dimensões altura e diâmetro da copa das árvores com o consumo de energia.

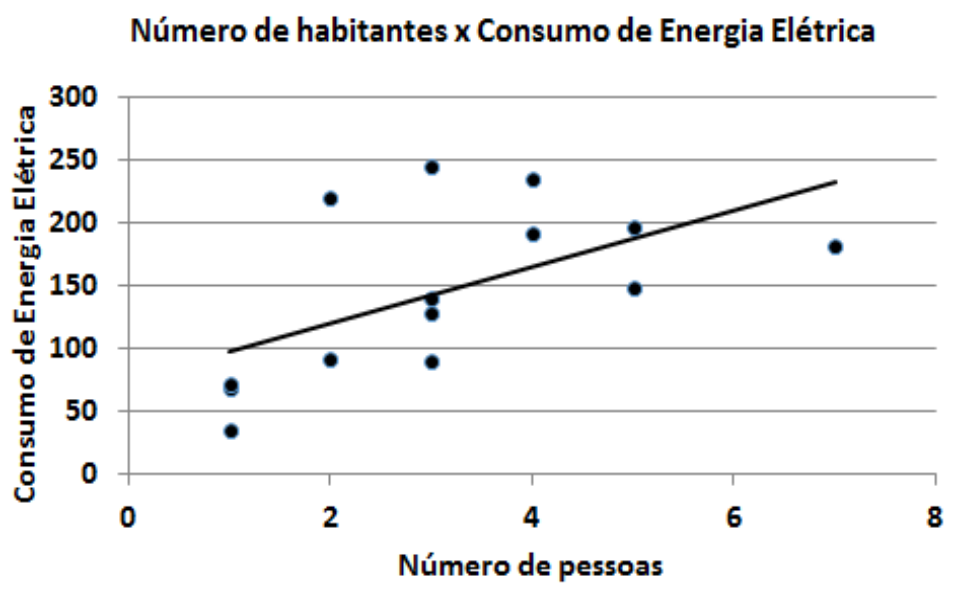

Figura 2. Gráfico de dispersão Número de pessoas x Consumo de energia elétrica Figure 2. Scatter plot Number of persons $x$ Power consumption

A situação ideal para comparação era a de que todas as residências possuíssem o mesmo número de moradores. Para tentar superar essa dificuldade, as residências foram divididas em duas classes: casas com dois ou menos moradores e casas com mais de dois moradores.

A Figura 3 apresenta dois gráficos de colunas sobre a média de consumo para as seguintes populações: casas com dois ou menos moradores $\mathrm{x}$ casas com mais de 2 moradores e casas voltadas para o leste $x$ casas voltadas para o oeste.

O exame da orientação da casa em relação ao sol se deve a suspeita de que as casas que têm as frentes voltadas para o poente têm temperatura do ar interna mais quente, provocando um maior consumo de energia elétrica mensal.

Um teste $\mathrm{F}$ indicou diferença altamente significativa entre as populações com dois ou menos moradores e com mais de dois moradores, com $p$-valor $=0,0053$. Baseado nesse resultado foi analisado o consumo de energia para casas com árvores na frente $\mathrm{x}$ casas sem árvores na frente para cada uma das populações.

$\mathrm{O}$ teste $\mathrm{F}$ também foi aplicado para comparar residências voltadas para o Leste $\mathrm{x}$ residências voltadas para o Oeste, o p-valor $=0,40$, o que indica que não existe diferença significativa entre as posições das casas em relação aos pontos cardeais. 

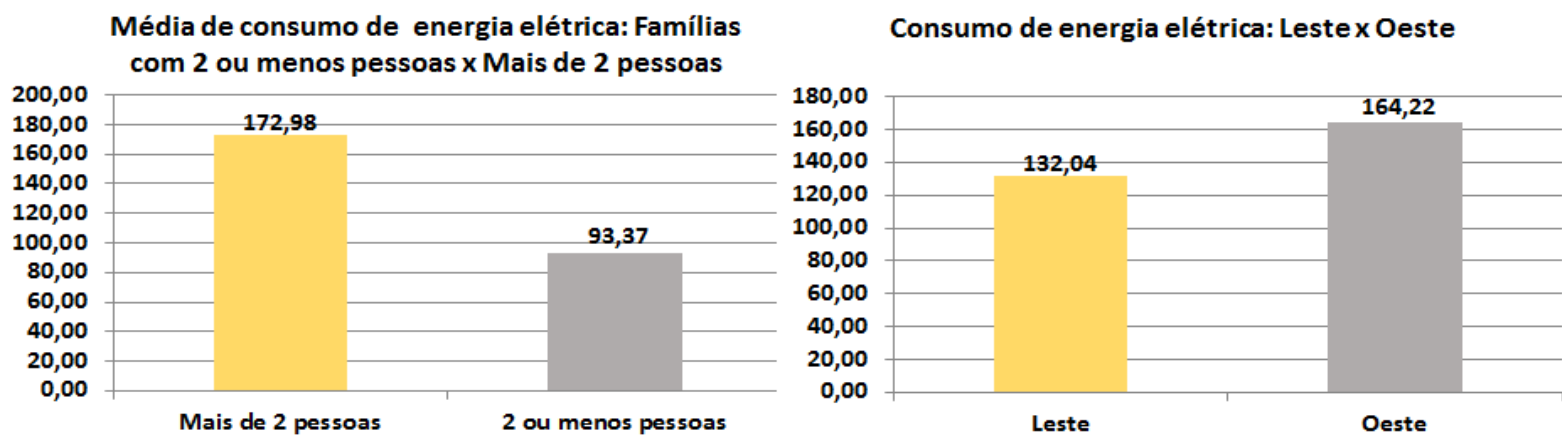

Figura 3. Média do consumo de energia para famílias com duas ou menos $\mathrm{x}$ mais de duas pessoas $\mathrm{e}$ para residências voltadas para o leste $x$ oeste

Figure 3. Energy consumption average for families with two or fewer $x$ more than two people and houses turned to the east $x$ west

\section{a) Ter ou Não ter árvore $x$ Consumo de energia elétrica}

A Figura 4 mostra as médias de consumo de energia elétrica para as populações: famílias com dois ou menos moradores e famílias com mais que dois moradores com e sem árvores na frente de casa.
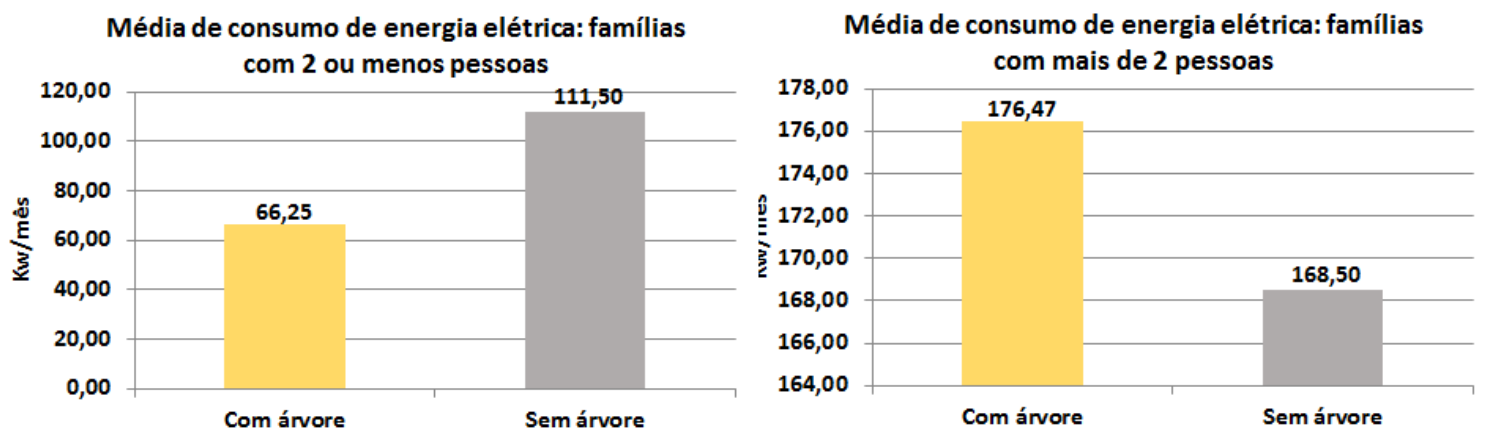

Figura 4. Média de consumo de energia elétrica de famílias com dois ou menos moradores e famílias com mais de dois moradores, ambas com e sem árvores em frente da casa

Figure 4. Electricity consumption average of families with two or fewer residents and families with more than two residents, both with and without trees in front of the house

O teste $\mathrm{F}$ foi aplicado para os dois casos: Para famílias com duas ou menos pessoas foi detectada diferença significativa apenas a $20 \%$, o comum é serem usados testes para 1 e $5 \%$. Existe, portanto, $80 \%$ de confiança de que as populações são diferentes, ou seja, famílias com duas ou menos pessoas consomem menos energia elétrica quando existe árvore em frente as suas residências. Para residências com dois ou menos moradores o consumo de energia elétrica é significativamente diminuído com a presença de árvore na calçada. Silva e Rosa (2004) verificaram o consumo de energia elétrica de diversos eletrodomésticos, as 
medições foram efetuadas no dia a dia de algumas casas. Eles verificaram que entre vários fatores que aumentam o consumo, um dos mais influentes é o número de residentes.

A árvore na calçada impede a incidência direta dos raios solares na parede frontal da casa. Diminuindo assim a temperatura do ar no interior da casa, o que por sua vez, diminui o tempo de uso dos aparelhos elétricos usados para amenizar o calor (ar condicionado e ventilador). O consumo de água gelada para beber também é diminuído, consequentemente a frequência com que o refrigerador é aberto diminui, e a abertura frequente do refrigerador é conhecidamente um forte fator de aumento do consumo energia elétrica.

Para a classe, mais que duas pessoas, não foi detectada diferença significativa, com o $p$-valor $=0,8317$. Portanto, é possível considerar que a presença ou ausência de árvores na frente da casa não altera o consumo de energia elétrica. Os fatores de diminuição do consumo também devem estar presentes nas residências com mais de dois moradores, porém nesse caso a influência do fator "pessoa" usando mais energia elétrica parece ter mascarado essa influência, de modo que o teste para os dados coletados não pode detecta-la.

\section{b) Altura da árvore x Consumo de energia elétrica}

A correlação entre a altura da árvore na calçada e o consumo de energia elétrica foi medida e forneceu um $r=-0,51$ para as residências com duas ou menos pessoas, indicando uma correlação mediana negativa. Para as residências com mais de duas pessoas $(r=0,02)$, o baixo valor da correlação indica que a altura da árvore não altera o consumo de energia elétrica para residências com mais de dois moradores.

O aumento da área de cobertura arbórea é fator de diminuição da temperatura do ar, que por sua vez melhora o conforto térmico. Lundgren e Silva (2013) realizaram o censo das árvores das calçadas em Serra Talhada - PE e verificaram que a correlação entre altura e diâmetro da copa foi $(r=0,97)$. A Figura 5 mostra o gráfico de dispersão e o modelo matemático da equação de regressão para a altura da árvore x consumo de energia, para as duas populações.

A equação de regressão que forneceu melhor ajuste para dois ou menos residentes $x$ altura da árvore foi $Y=105,5(E)^{0,14 X}$ com $\mathrm{R}^{2}=0,338$ e modelo exponencial e e para mais que dois residentes $\mathrm{x}$ altura da árvore foi $Y=-3,3489 X^{2}+19,913 X+168,42 \mathrm{com} \mathrm{R}^{2}=0,0365 \mathrm{e}$ modelo polinomial de grau 2.

O valor mediano do $\mathrm{R}^{2}$ inviabiliza o uso da equação na previsão do consumo de energia usando como variável independente a altura da árvore em residências com dois ou menos moradores e o mesmo acontece para residências com mais de dois moradores. 
A estimativa do consumo de energia elétrica usando as equações de regressão em que a única variável independente foi a altura da árvore, não forneceu resultados satisfatórios, pois, vários fatores independentes ou correlacionados entre si, influem no consumo de energia elétrica de uma residência, e esses fatores não foram levados em consideração no presente trabalho.

A CEPEL (2014) fornece uma lista de procedimentos associados aos fatores que influenciam no consumo de energia elétrica em uma residência. Os procedimentos são: não deixar luzes acesas desnecessariamente, pintar paredes com cores claras, durante o dia deixar cortinas e portas abertas, globos e lustres sempre limpos, filtros de ar-condicionado sempre limpos, usar o termostato do ar condicionado, acumular roupas para lavar e passar ferro, manter a geladeira sempre bem vedada, em dias secos colocar toalhas molhadas em vez de umidificadores elétricos, desligar aparelhos elétricos da tomada (stand-by), fogão e geladeira não devem estar próximos, vedação da geladeira em bom estado, regular a geladeira conforme a estação do ano.
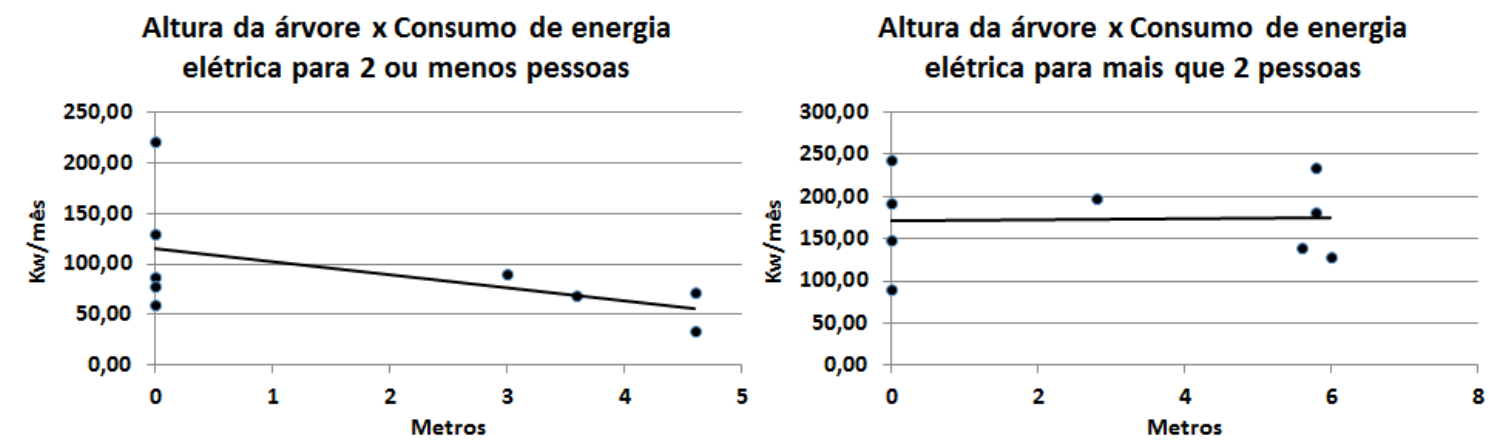

Figura 5. Gráfico de dispersão da Altura x Consumo para casas com dois ou menos residentes e casas com mais de dois residentes

Figure 5. Scatter plot height $\mathrm{x}$ consumption for homes with two or fewer residents and households with more than two residents

\section{c) Diâmetro da copa x Consumo de energia elétrica}

A Figura 6 mostra o gráfico de dispersão e o modelo matemático para a relação diâmetro da copa x consumo de energia elétrica para as residências com dois ou menos moradores e com mais de dois moradores.

A equação de regressão que melhor se ajustou aos dados do diâmetro da copa $x$ consumo de energia elétrica para residências com dois ou menos moradores foi $Y=102,17 E^{-0,15 X}$ com o $\mathrm{R}^{2}=0,297$. Com modelo exponencial, a correlação entre o diâmetro da copa e o consumo de energia elétrica foi $r=-0,4765$. 
Para as residências com mais de dois moradores a correlação foi $r=0,0941$ indicando que a correlação é próxima de zero, ou seja, quase que inexiste. Para esse caso é desnecessária a construção de equação de regressão, a mesma não traz nenhuma informação relevante.

A Tabela 1 apresenta os modelos de regressão testados para cada uma das duas classes populacionais com e sem árvores na frente de casa.

É possível observar que para as residências com dois ou menos moradores o modelo linear conseguiu um coeficiente de determinação maior do que para as residências com mais de dois moradores, contudo, nenhum dos três modelos testados conseguiu um $\mathrm{R}^{2}$ razoável, todos foram muito baixos e próximos a zero, indicando, que as equações não fornecem estimativas confiáveis.

Para os modelos testados para a relação número de moradores $x$ consumo de energia elétrica, o melhor modelo foi o polinomial de segunda ordem.
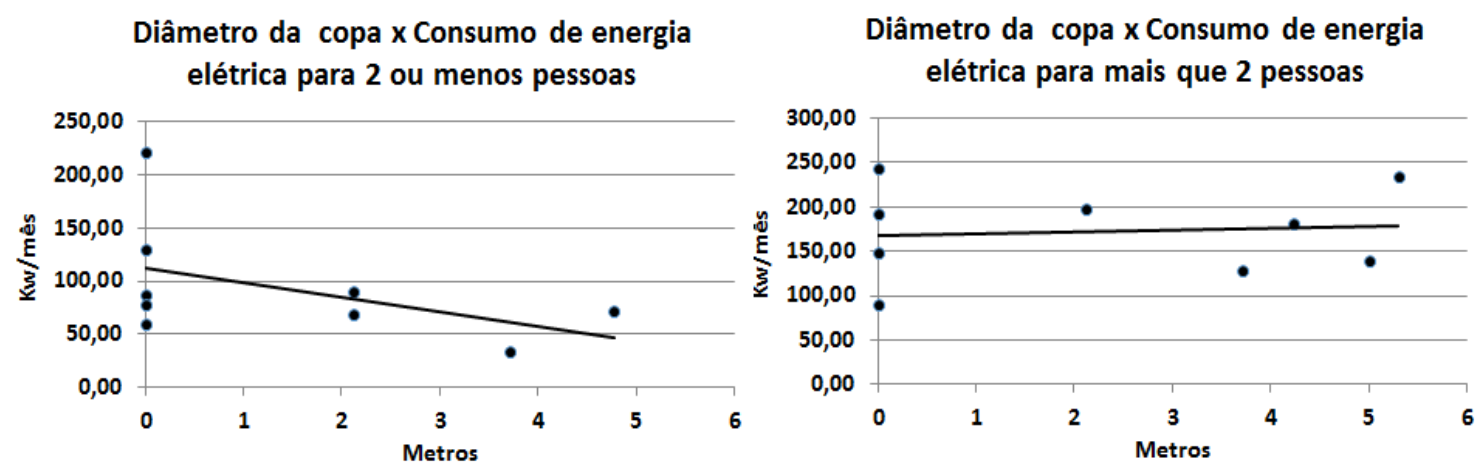

Figura 6. Gráfico de dispersão do Diâmetro da copa x Consumo de energia elétrica para casas com dois ou menos e casas com mais de dois residentes

Figure 6. Scatter plot Cup diameter $x$ Consumption of electricity for homes with two or fewer residents and more than two residents

O modelo linear, $Y=a X+b$, em que "a" é o coeficiente de inclinação da reta, " $b$ " é o intercepto, $Y$ é a variável dependente e $X$ a variável independente, apesar de ter fornecido os piores valores de $\mathrm{R}^{2}$ para as três populações testadas tem a propriedade de permitir um entendimento direto de seus resultados, pois, o parâmetro "a" mostra, que para cada aumento unitário da variável independente, ocorre exatamente um aumento de dimensão "a" para a variável dependente.

$\mathrm{Na}$ relação número de pessoas $\mathrm{x}$ consumo de energia elétrica é possível ver na Tabela 1 que para cada pessoa a mais que mora na residência há um aumento de aproximadamente $24 \mathrm{kw} /$ mês (valor do parâmetro $a=23,67$ ) e para cada aumento de 1 metro na altura da árvore há uma diminuição de aproximadamente $13 \mathrm{kw} / \mathrm{mês}$ (valor do parâmetro $a=$ -12,87) e para cada aumento de 1 metro no diâmetro da copa da árvore há uma diminuição de 
aproximadamente $14 \mathrm{kw} /$ mês (valor do parâmetro $a=-13,89$ ), considerando as residências com dois ou menos moradores.

Para as residências com mais de duas pessoas, a altura e o diâmetro da copa das árvores perdem grande parte de sua influência, pois o número de residentes passa a ser o fator preponderante para o consumo.

É importante ressaltar que as árvores na cidade de Serra Talhada costumam ser pequenas em altura e na dimensão das copas. Lundgren, Silva e Almeida (2013) afirmam que a média dos diâmetros de copa das árvores nas calçadas da cidade não ultrapassa 6 metros e que a altura média das árvores é menor que 4 metros.

Tabela 1. Parâmetros das equações de regressão. $N P=$ Número de pessoas na residência, $C O=$ consumo de energia elétrica, $\mathrm{AL}$ = altura da árvore, $\mathrm{CP}$ = diâmetro da copa da árvore

Table 1. Parameters of the regression equations. NP $=$ number of people in the residence, $C O=$ electricity consumption, $\mathrm{AL}=$ tree height, $\mathrm{CP}=$ tree canopy diameter

\begin{tabular}{|c|c|c|c|c|c|c|c|c|c|c|c|}
\hline \multirow{3}{*}{ Classes residenciais } & \multirow{3}{*}{ Relação } & \multicolumn{10}{|c|}{ Modelos } \\
\hline & & \multicolumn{3}{|c|}{ Linear } & \multicolumn{3}{|c|}{ Exponencial } & \multicolumn{4}{|c|}{ Polinomial } \\
\hline & & $\mathrm{a}$ & $\mathrm{b}$ & $\mathrm{R}^{2}$ & $a$ & $\mathrm{~b}$ & $\mathrm{R}^{2}$ & a & $b$ & $\mathrm{C}$ & $\mathrm{R}^{2}$ \\
\hline Todos & $\mathrm{NP} \times \mathrm{CO}$ & 23,67 & 66 & 0,36 & 65 & 0,21 & 0,40 & $-5,90$ & 66 & 9 & 0,45 \\
\hline \multirow{2}{*}{2 ou menos } & $\mathrm{AL} \times \mathrm{CO}$ & $-12,87$ & 116 & 0,26 & 106 & $-0,14$ & 0,34 & $-2,55$ & -2 & 115 & 0,27 \\
\hline & $\mathrm{CP} \times \mathrm{CO}$ & $-13,89$ & 113 & 0,23 & 102 & $-0,15$ & 0,30 & 3,54 & -29 & 116 & 0,25 \\
\hline \multirow{2}{*}{ Maior que 2} & $\mathrm{AL} \times \mathrm{CO}$ & 0,42 & 172 & 0,00 & 162 & 0,01 & 0,01 & $-3,35$ & 20 & 168 & 0,04 \\
\hline & $\mathrm{CP} \times \mathrm{CO}$ & 2,05 & 168 & 0,01 & 159 & 0,02 & 0,02 & 2,79 & -11 & 171 & 0,03 \\
\hline
\end{tabular}

\section{CONCLUSÃO}

Casas com frente menor que cinco metros, sem terraços ou varandas, de famílias de baixo poder aquisitivo com menos que três moradores, diminuem o consumo de energia elétrica quando possuem árvore na calçada.

A altura da árvore foi inversamente correlacionada com o consumo de energia elétrica.

A copa da árvore foi inversamente correlacionada com o consumo de energia elétrica.

A presença da árvore na calçada as casas não mostrou influenciar no consumo de energia elétrica quando as residências possuem mais que dois moradores.

Não foi detectada diferença no consumo de energia entre as casas voltadas para o leste ou oeste. 


\section{REFERÊNCIAS}

ASSIS, J. M. O. de; SILVA, R. F. da; SOUZA, W. S. da; ALEXANDRE IRMÃO, R.; CORREIA, A. M. Tendências Climáticas Observadas no Período Chuvoso no Sertão de Pernambuco (Observed Climate Trends in Rainy Season in the Sertão of Pernambuco). Revista Brasileira de Geografia Física, Recife - PE, v. 6, n. 2, p. 211-222, 2013.

CENTRO DE PESQUISAS DE ENERGIA ELÉTRICA (CEPEL) - Eletrobrás. Setor Residencial Dicas de Economia de Energia. Disponível em: < http://www.cate.cepel.br/setatuac/residenc/dicas_res.htm>. Acesso em: 13 set. 2014.

FEITOSA, S. M. R; GOMES, J. M. A.; MOITA NETO, J. M; ANDRADE, C. S. P. de. Consequências da urbanização na vegetação e na temperatura da superfície de TeresinaPiauí. Sociedade Brasileira de Arborização Urbana, Piracicaba - SP. v. 6, n. 2, p. 58-75, 2011.

INSTITUTO BRASILEIRO DE GEOGRAFIA E ESTATÍSTICA (IBGE). Censo 2010. Disponível em: <http://cidades.ibge.gov.br/xtras/perfil.php?codmun=261390>. Acesso em: 31 ago. 2014.

KURIHARA, D. L.; IMAÑA - ENCINAS, J.; PAULA, J. E. de. Levantamento da arborização do campus da Universidade de Brasília. Cerne, Lavras - MG, v. 11, n. 2, p. 127-136, 2005.

LUNDGREN, W. J. C.; SILVA, L. F. da. Correlação entre índices das árvores e classes sociais na cidade de Serra Talhada - PE. Revista da Sociedade Brasileira de Arborização Urbana. Piracicaba - SP, v.8, n.4, p 107-124, 2013.

LUNDGREN, W. J. C.; SILVA, L. F. da; ALMEIDA, A. Q. de. Influência das espécies exóticas arbóreas urbanas na área de cobertura da cidade de Serra Talhada - PE. Revista da Sociedade Brasileira de Arborização Urbana, Piracicaba - SP, v.8, n.3, p 96-107, 2013.

MARTINE, G. "O lugar do espaço na equação população/meio-ambiente". In Revista Brasileira de Estudos de População. São Paulo: ABEP. v. 24, n. 2, p. 181-190, 2007.

NOGUEIRA, C. E. C.; SIQUEIRA, J. A. C.; SOUZA, S. N. M. de; NIEDZIALKOSKI, R. K.; PRADO, N. V. do. Avaliação do conforto térmico nas residências convencional e inovadora do "Projeto CASA", Unioeste, Campus de Cascavel. Acta Scientiarum. Technology, Maringá SC. v. 34, n. 1, p. 3-7, 2011.

ROPPA, C.; FALKENBERG , J. R.; STANGERLIN , D. M.; BRUN , F. G. K.; BRUN , E. J.; LONGHI, S. J. Diagnóstico da percepção dos moradores sobre a arborização urbana na Vila Estação Colônia - bairro Camobi, Santa Maria - RS. Revista da Sociedade Brasileira de Arborização Urbana, Piracicaba - SP, v. 2, n. 2, p. 11-30, 2007.

SHIU, A; LAM, P-L. Electricity consumption and economic growth in China. Energy policy, v. 32, n. 1, p. 47-54, 2004.

SILVA, A. C. M. da; ROSA, L. P. Análise condicionada da demanda com correção de heterocedasticidade. In: XXXVI SIMPÓSIO BRASILEIRO DE PESQUISA OPERACIONAL, 2004, São João Del Rey - MG. Anais do XXXVI SBPO, São João Del Rey, 2004. p. 739 749. 
SOUZA, L. C. L.; POSTIGO, C. P.; OLIVEIRA, A. P.; NAKATA, C. M. Urban heat islands and electrical energy consumption. International Journal of Sustainable Energy. New York - NY. v. 28, n. $1-3$, p. 113-121, 2009.

SUPERINTENDÊNCIA DO DESENVOLVIMENTO DO NORDESTE (SUDENE). Dados pluviométricos mensais do Nordeste - Estado de Pernambuco. Recife, 1990. 363p

VELASCO, G. D. N. LIMA, A. M. L. P.; COUTO, H. T. Z. do; SILVA FILHO, D. F. da; POLIZEL, J. L.. Avaliação de método de questionário para estudo da relação entre presença e uso de aparelhos de refrigeração, arborização viária e consumo de energia elétrica. Revista Árvore, Viçosa - MG. v. 35, n. 3, supl. 1, p. 641-648, 2011. 